\title{
Determinação de hemogregarina em Boa constrictor constrictor mantidos em cativeiro ${ }^{1}$
}

\author{
Monique A. Luz ${ }^{2}$, Andre M.C. Meneses ${ }^{2 *}$, Carla C.G. de Moraes ${ }^{3}$, Larissa dos S. \\ Seixas $^{2}$, Dennis J. da S. Lima ${ }^{2}$, Luis A.A. dos S. Ruffeil ${ }^{4}$, Paulo H.G. de Castro ${ }^{4}$ \\ e Antonio M. Costa ${ }^{5}$
}

\begin{abstract}
Luz M.A., Meneses A.M.C., Moraes C.C.G., Seixas L.S., Lima D.J.S., Ruffeil L.A. A.S., Castro P.H.G. \& Costa A.M. 2012. [Determination of hemogregarine in Boa constrictor constrictor kept in captivity.] Determinação de hemogregarina em Boa constrictor constrictor mantidos em cativeiro. Pesquisa Veterinária Brasileira 32(8):781-785. Laboratório de Patologia Clinica Veterinária, Instituto da Saúde e Produção Animal, Universidade Federal Rural da Amazônia, Av. Presidente Tancredo Neves 2501, Bairro Montese, Belém, PA 66077-530, Brasil. E-mail: andre.meneses@ufra.edu.br

We aimed to determine hemogregarines presence in snakes of the Boidae family kept in captivity in Pará (PA), Brazil, and to relate it with sex, clinical and hematological and ectoparasitism. This study had authorization from Sisbio/IBAMA to be done. Nineteen Boa constrictor snakes were used, belonging to the "Museu Paraense Emílio Goeldi" (Belém/ PA) and "Xerimbabo Farm" (Santo Antônio do Tauá/PA). Blood smears were examined with 400x magnification, while the parasitemia percentage was determined by counting 550 red blood cells with $1000 x$ magnification. From the snakes studied $(n=19)$, nine were parasitized $(47.36 \%)$ and there was no correlation between hemogregarines presence, sex, clinical and hematological changes. Hemoparasitosis correlation was detected only with the ectoparasites presence; however further studies are needed to determine the real hemogregarines prevalence in snakes kept in captivity in Pará, since there is a huge gap of data in the veterinary specialized literature about the fauna of the Amazon region.
\end{abstract}

INDEX TERMS: Boa constrictor constrictor, Boidae, blood parasites, hemogregarines, reptiles, snakes, captivity.

RESUMO.- 0 presente estudo teve como objetivo determinar a presença de hemogregarina em boídeos mantidos em cativeiro no Estado do Pará, bem como, relacionar a hemoparasitose com pre-disposição sexual, alterações clínicas e hematológicas e a presença de ectoparasitos. Esta pesquisa teve autorização do Sistema de Autorização e Informação em Biodiversidade do Instituto Brasileiro do Meio Ambiente e dos Recursos Naturais Renováveis para

\footnotetext{
${ }^{1}$ Recebido em 14 de novembro de 2011.

Aceito para publicação em 20 de março de 2012.

${ }^{2}$ Laboratório de Patologia Clinica Veterinária, Instituto da Saúde e Produção Animal, Universidade Federal Rural da Amazônia, Av. Presidente Tancredo Neves 2501, Bairro Montese, Belém.PA 66077-530, Brasil. *Autor para correspondência: andre.meneses@ufra.edu.br

${ }^{3}$ Faculdade de Medicina Veterinária, Universidade Federal do Pará, Campus de Castanhal, Rua Maximino Porpino da Silva 1000, Pirapora, PA 68740-080, Brasil.

${ }^{4}$ Sitio Xerimbabo, Rua do Ficado, Km 12, Santo Antônio do Tauá, PA 68786-000, Brasil.

${ }^{5}$ Museu Paraense Emilio Goeldi, Av. Governador Magalhães Barata 376, Belém, PA 66040-170.
}

ser realizado. Utilizaram-se 19 serpentes da família Boidae mantidas em cativeiro, pertencentes ao "Museu Paraense Emilio Goeldi" (Belém/PA) e "Sítio Xerimbabo" (Santo Antônio do Tauá/PA). A pesquisa de hemogregarina foi realizada em esfregaços sanguíneos examinados no aumento de $400 x$, enquanto que a parasitemia foi determinada contando-se 550 hemácias em aumento de 1000x. Do total de animais estudados $(\mathrm{n}=19)$, nove encontraram-se parasitados $(47,36 \%)$, não havendo correlação entre presença de hemogregarina, pré-disposição sexual, alterações clínicas e hematológicas nas serpentes hospedeiras. A correlação da hemoparasitose foi detectada apenas quanto à presença de ectoparasitas nas serpentes, no entanto, estudos adicionais são necessários para verificar a prevalência de hemogregarinas em animais mantidos em cativeiro no Estado do Pará, visto que, existe grande lacuna de dados na literatura veterinária especializada no que diz respeito à fauna da região amazônica.

TERMOS DE INDEXAÇÃO: Boa constrictor constrictor, Boidae, hemoparasitos, hemogregarina, répteis, serpentes, cativeiro. 


\section{INTRODUÇÃo}

Os animais silvestres estão expostos a inúmeros agentes infecciosos e parasitários, dentre eles os hemoparasitos, que são transmitidos por vetores hematófagos (Leal et al. 2007). Sua presença é considerada um achado acidental em algumas espécies, no entanto, podem provocar doenças como, anemia hemolítica. Dentre os hemoprotozoários comuns em répteis estão hemogregarinas, tripanossomos e Plasmodium (Thrall et al. 2007).

Boa constrictor constrictor, subespécie muito difundida nos centros de pesquisas, criadouros conservacionistas, zoológicos e outros do gênero, especializados em répteis, é uma serpente da família Boidae, onde estão classificadas as maiores serpentes do mundo (Vanzolini et al. 1980). A manutenção desta espécie em cativeiro requer manejo apropriado e dieta balanceada, embora algumas medidas preventivas adotadas não sejam adequadas e/ou suficientes para assegurar a saúde das serpentes (Hoge \& Federsoni 1981). A despeito disso, o cativeiro, por vezes, proporciona condições ideais para que a transmissão e perpetuação do ciclo de vida dos hemoparasitos aconteçam (O'Dwyer et al. 2003).

As hemogregarinas são os parasitos intra-eritrocitários mais comumente observados em serpentes cativas (Garcia-Navarro \& Pachaly 1994). 0 termo hemogregarina é usado para descrever coletivamente parasitos sanguineos pertencentes às subordens Adeleiina e Eimeriina do Filo Apicomplexa (Levine et al. 1980).

Em Eimeriina, a esporogonia e a merogonia ocorrem no hospedeiro vertebrado e o hospedeiro invertebrado serve apenas como vetor mecânico para a transmissão dos parasitos. Por outro lado, na subordem Adeleiina, do qual fazem parte os gêneros Hepatozoon, Haemogregarina e Karyolysus, a esporogonia ocorre no vetor e a merogonia no hospedeiro vertebrado (Lane \& Mader 1996), tendo maior destaque os dois primeiros gêneros_(Jacobson 1986).

Estes protozoários são encontrados no interior dos eritrócitos do animal parasitado e seu ciclo está ligado a artrópodes hematófagos (Garcia-Navarro \& Pachaly 1994), onde para o gênero Hepatozoon, a esporogônia ocorre na hemocele desses vetores, com a formação de grandes oocistos, com centenas de esporocistos contendo esporozoítos no seu interior (Moço 2008). 0 desenvolvimento merogônico e gametogônico ocorre nos órgãos internos, como fígado e pulmões, do hospedeiro vertebrado após a ingestão de um hospedeiro definitivo invertebrado ou um hospedeiro vertebrado intermediário infectado (Smith 1996).

Para o gênero Haemogregarina, a esporogonia ocorre no estômago do vetor, os oocistos não possuem esporocistos e sua transmissão é feita através da inoculação de esporozoítas via saliva (Moço 2008).

Um grande número de hospedeiros vertebrados tem sido encontrado, incluindo as aves, várias espécies de mamíferos, crocodilianos, lagartos, cobras, tartarugas e, anuros (Desser et al., 1995, Smith, 1996).

Muitos invertebrados foram descritos como vetores e hospedeiros definitivos, incluindo os carrapatos, mosquitos, moscas, piolhos, pulgas e sanguessugas (Clark et al. 1973, Göbel \& Krampitz 1982, Telford Jr 1984).
Os hematozoários deste gênero parecem ser extremamente adaptados, pois causam pouca ou nenhuma alteração clínico-patológica em seus hospedeiros naturais (Moço 2008), porém, casos de anemia hemolítica podem ser observados (Garcia-Navarro \& Pachaly 1994, Campbell 1996).

0 potencial patogênico desta parasitose em hospedeiros não naturais, pode alcançar proporções significativas em condições de cativeiro, quando não há um rígido controle parasitológico, justificando-se a necessidade de um bom diagnóstico para evitar a disseminação da doença dentro do criadouro. 0 diagnóstico é feito pela técnica do esfregaço sanguíneo (Campbell 1996, O’Dwyer et al. 2003), mas, atualmente, diagnósticos mais modernos incluem a avaliação do sangue pela técnica de Reação em Cadeia da Polimerase ( PCR) (Wosniak et al. 1994).

Portanto, o presente estudo propôs determinar a presença de hemogregarina em boídeos Amazônicos mantidos em cativeiro, investigando o hemoparasito em serpentes sadias e enfermas, para determinar se há correlação entre a hemoparasitose e o sexo, alterações clínicas e hematológicas e a presença de ectoparasitos nas serpentes hospedeiras. Este estudo virá a contribuir com a parasitologia veterinária de animais silvestres, que possui poucos dados em termos de Amazônia, especialmente em se tratando de hemogregarinas e da sanidade de animais mantidos em cativeiro no Estado do Pará.

\section{MATERIAL E MÉTODOS}

A presente pesquisa teve autorização do Ministério do Meio Ambiente (MMA), Instituto Brasileiro do Meio Ambiente e dos Recursos Naturais Renováveis (IBAMA), Instituto Chico Mendes de Conservação da Biodiversidade (ICMBio), Sistema de Autorização e Informações em Biodiversidade (Sisbio), sob o número 16851-1

Foram utilizadas 19 serpentes de ambos os sexos da espécie Boa constrictor constrictor mantidas em cativeiro no Estado do Pará_sendo 10 provenientes do Sítio Xerimbabo, criatório comercial com autorização do IBAMA/MMA, localizado no município de Santo Antônio do Tauá/Pará (latitude 01ํ0'07" sul e longitude 480 $07^{\prime} 46^{\prime \prime}$ oeste), e outras nove serpentes pertencentes ao Museu Paraense Emilio Goeldi de Belém/PA (latitude $01^{\circ} 27^{\prime} 21^{\prime \prime}$ sul e longitude $48^{\circ} 30^{\prime} 16^{\prime \prime}$ oeste).

Os animais eram mantidos em cativeiro coletivo em viveiro aberto (Sítio) ou em caixas individuais (Museu) e todos eram alimentados com filhotes de ratos, oferecidos em intervalos de três dias.

Após contenção física, foi realizado exame clínico dos animais de acordo com Hiinarejos et al. (2007), da direção cranial para a caudal, incluindo inspeção da cavidade oral, dos orifícios nasais e da membrana ocular, palpando-se todo o corpo dos animais e finalizando com uma inspeção da cloaca. A pele e a superfície interior das escamas foram examinadas em busca de ácaros, problemas de muda, trauma ou infecções.

Em seguida realizou-se a colheita de sangue por cardiocentese de acordo com o recomendado por Thrall et al. (2007), sendo este um procedimento muito empregado para este fim em serpentes.

Foram colhidos $2 \mathrm{~mL}$ de sangue vertidos em tubos de vidro contendo heparina sódica, anticoagulante preconizado para exame hematológico de répteis, uma vez que há relatos sobre hemólise associado com o uso de ácido etilenodiaminoteraacético (EDTA) para esses animais (Kolesnikovas et al. 2007). 0 material foi mantido resfriado $\left(4-8^{\circ} \mathrm{C}\right)$, até seu processamento, que foi realizado no mesmo dia da colheita, no Laboratório de Patologia 
Clínica Veterinária do Instituto da Saúde e Produção Animal da Universidade Federal Rural da Amazônia.

As contagens totais de eritrócitos e leucócitos foram realizadas a partir da diluição de sangue 1:200 em solução isotônica de Natt-Herrick, utilizando a Câmara de Neubauer. 0 método empregado foi diferente daqueles utilizados para mamíferos, pois em répteis todas as células são nucleadas e devem ser observadas e contadas em uma única fase (Kolesnikovas et al. 2007). Em seguida, a pesquisa dos hemoparasitos foi realizada em esfregaços corados por corantes hematológicos instantâneos da marca Panótico ${ }^{\circledR}$.

De acordo com Desser et al. (1995), a identificação genérica depende da natureza do desenvolvimento esporogônico no vetor, portanto, como no presente estudo não se observou o ciclo biológico neste, chamou-se de hemogregarinas os parasitos detectados nos esfregaços sanguíneos. Estes foram identificados pela presença de gametócitos intracitoplasmáticos nos eritrócitos, apresentando formato de "linguiça" e núcleo grande central ou periférico (Telford Jr 2000, Thrall et al. 2007).

A classificação da parasitemia obedeceu aos critérios propostos por De Biase et al. (1989), onde em um aumento de 1000x se considerava esfregaços apresentando até um parasito para cada três campos ópticos como infecção leve (+); encontro entre o limite máximo de infecção leve até três parasitos por campo óptico como infecção média (++) e acima do índice máximo da infecção média como uma infecção grave: $(+++)$.

0 teste Qui-quadrado $\left(x^{2}\right)$ foi utilizado para verificar a associação entre o sexo, sanidade, presença de ectoparasitos e animais parasitados por hemogregarinas e na avaliação hematológica utilizou-se a determinação da amplitude de variação de um desvio padrão. 0 nível de significância adotado foi de $5 \%$.

\section{RESULTADOS}

Das 19 serpentes estudadas (11 eram machos e 8 fêmeas). Deste total, nove apresentaram a infecção por hemogregarina $(47,37 \%)$, sendo sete machos $(77,78 \%$ dos infectados $)$ duas fêmeas $(22,22 \%$ dos infectados).

Dez das 19 serpentes do presente estudo encontravam-se clinicamente doentes, considerando-se também como clinicamente doentes aquelas que se encontravam com ectoparasitos.

Cinco destes animais clinicamente doentes encontravam-se parasitados por hemogregarina, sendo que uma serpente apresentava estomatite e anorexia, outra apresentava nódulos pelo corpo, outra com quadro de desidratação e presença de ectoparasitos (carrapatos) e outras duas apenas com presença de carrapatos. Dentre as serpentes clinicamente sadias ( $n=9)$, em quatro observou-se positividade para hemogregarina nos esfregaços sanguíneos destas.

Após análise estatística dos dados $(\mathrm{p}<0,05)$, concluiu-se que os desvios não foram significativos quanto ao sexo e a sanidade dos animais. Portanto, os indivíduos reagiram do mesmo modo, não havendo, na presente pesquisa, influência desses dois fatores sobre o resultado. Assim sendo, neste trabalho, a positividade independe do sexo e da sanidade das serpentes.

Quanto à presença de ectoparasitos, a análise estatística, com mesmo nível de significância $(\mathrm{p}<0,05)$, mostrou desvios significativos, ou seja, os indivíduos reagiram de modo diferente, sendo a positividade para hemogregarina no presente estudo, dependente da presença de ectoparasitos nas serpentes.
Considerando-se esses valores de referência, o hemograma das serpentes positivas para hemogregarina na presente pesquisa, encontrou-se dentro da amplitude normal de variação de um desvio padrão, portanto não havendo correlação entre a parasitemia e alterações hematológicas.

Cinco serpentes apresentaram infecção classificada como leve $(+)$, duas apresentaram infecção média $(++)$ e outras duas infecção grave $(++++)$, seguindo a classificação de De Biase et al. (1989).

A serpente que se encontrava com estomatite e anorexia, assim como as duas que se encontravam apenas com ectoparasitos, apresentaram o grau de infecção classificado como leve (Fig.1). As outras duas serpentes com infecção leve bem como as duas com infecção média (Fig.2) não se encontravam clinicamente doentes, mesmo quando classificadas desta forma devido à presença de ectoparasitos.

A serpente que apresentava nódulos pelo corpo e aquela que se encontrava desidratada e com carrapatos, foram as que apresentaram grau de infecção classificado como grave (Fig.3)

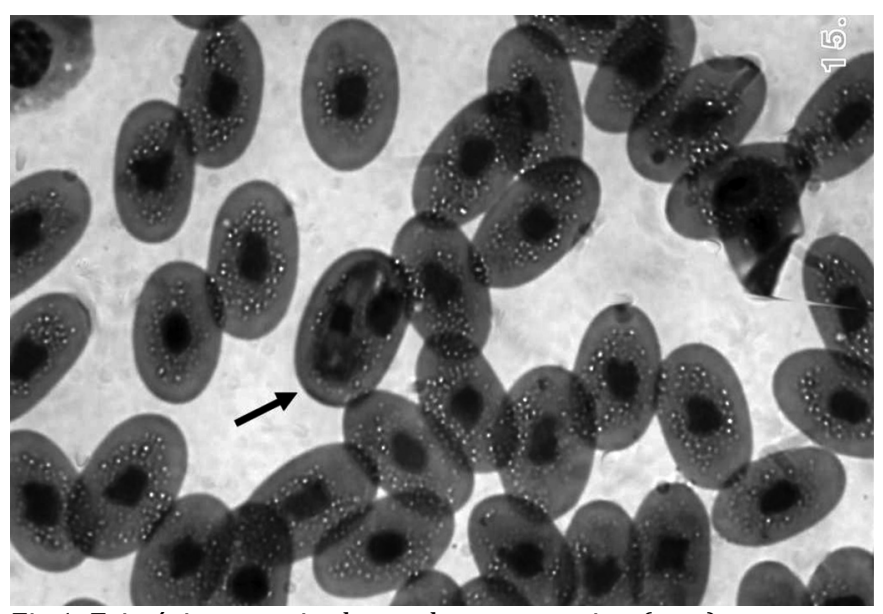

Fig.1. Eritrócito parasitado por hemogregarina (seta) em serpente com um grau de parasitemia leve (um parasito para cada três campos ópticos). HE, 1000x.

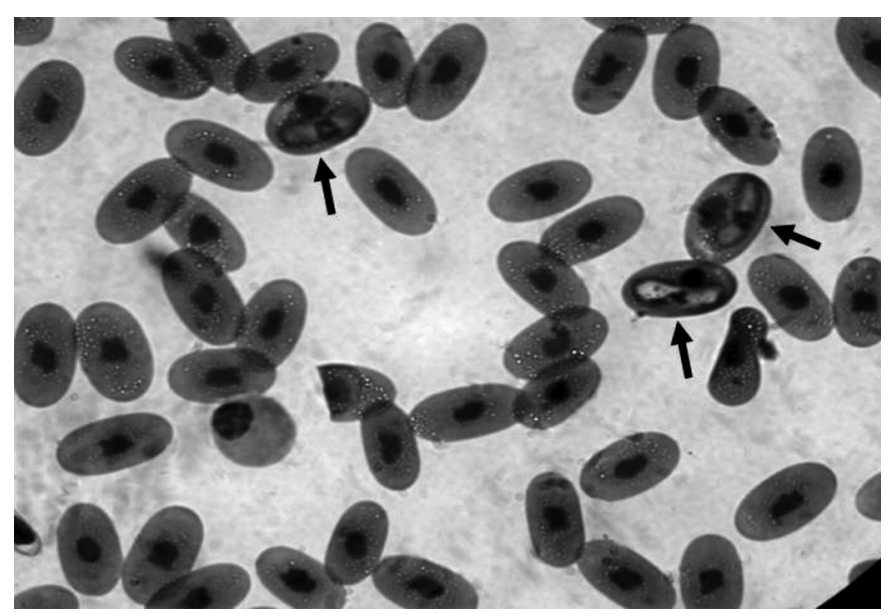

Fig.2. Eritrócitos parasitados por hemogregarinas (setas) em serpente com um grau de parasitemia médio (encontro entre limite máximo de infecção leve até três parasitos por campo óptico). HE, 1000x. 


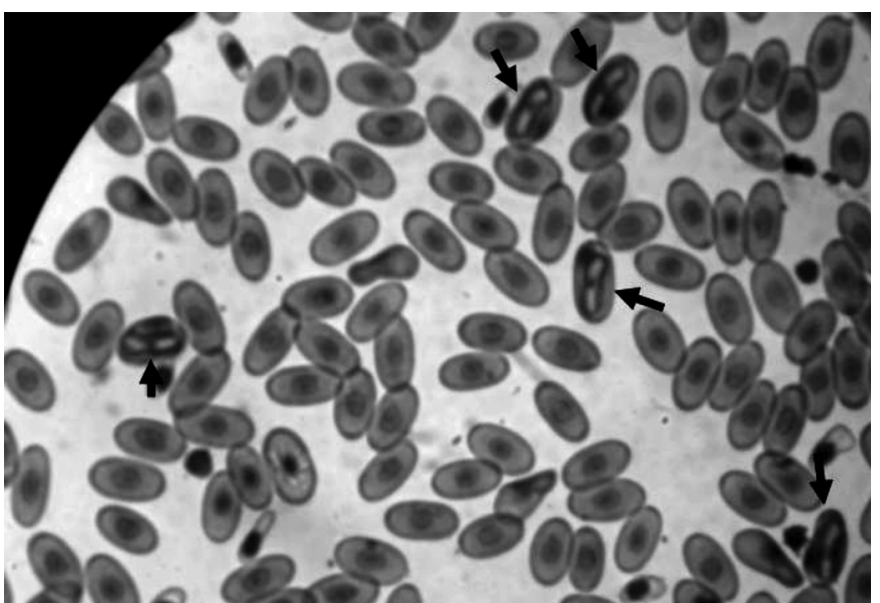

Fig.3. Eritrócitos parasitados por hemogregarinas (setas) em serpente, com grau de parasitemia grave (mais de três parasitos por campo óptico). HE, 1000x.

\section{DISCUSSÃO}

Os resultados desta pesquisa diferem daqueles encontrados por O'Dwyer et al. (2003), onde estudando a prevalência de Hepatozoon sp. em serpentes recém-capturadas no Brasil, encontraram uma positividade menor que a do presente estudo, o equivalente a $38,9 \%$ entre as 18 serpentes da espécie Boa constrictor amarali estudadas, sendo a infecção mais predominante entre as fêmeas_(57,1\% contra $14,3 \%$ ). Vale ressaltar que o presente estudo foi realizado em condições completamente diferenciadas do estudo anterior, pois se trabalhou na Região Amazônica e utilizando-se a subespécie Boa constrictor constrictor, que, segundo Cherubini et al. (2003), é a única encontrada nesta região. De acordo com relatos de Machado (2005), o local e o clima bem como a espécie, parecem influenciar no achado ou não de hemogregarina nas serpentes. Como a pesquisa foi realizada na região tropical, o resultado com alto número de serpentes positivas era esperado.

Machado (2005) descreve ainda que, a manutenção desses animais em cativeiro deve ser considerada como fator importante para a presença ou não do parasito, uma vez que as serpentes mantidas neste regime de criação possuem diferenças em relação àqueles que ainda se encontram na natureza, e ainda, que situações como ambiente, confinamento, alimentação, estresse e o convívio próximo com muitos animais acabam promovendo alterações imunológicas e fisiológicas, bem como favorecendo a transmissão de parasitos.

Goulart (2004) observou períodos de intensa parasitemia por hemogregarinas juntamente com quadros de estomatites em serpentes, sem causa predisponente, não se sabendo se a parasitemia leva à infecção bacteriana, ou se a queda da imunidade propicia a instalação dos dois quadros.

Das duas serpentes que se apresentavam com estomatite no presente estudo, apenas uma apresentou-se parasitada por hemogregarina, não sendo possível determinar esta correlação.

A positividade para hemogregarina em quatro serpentes clinicamente saudáveis vai de acordo com a literatura que cita que na maioria dos casos de infecção por hemogregarina, não se verificam sinais clínicos de doença (Garcia-Navarro \& Pachaly 1994, Smith 1996, Thrall et al. 2007).

Quanto à influência do sexo na presença de hemogregarina, os resultados desta pesquisa diferem dos achados de O'Dwyer et al. (2003), que observaram maior frequência da infecção entre as fêmeas (57,1\% contra $14,3 \%)$, mostrando uma predisposição sexual na referida pesquisa.

A presença de ectoparasitos como um fator determinante para a infecção por hemogregarinas nas serpentes do presente estudo, pode ser explicada pela acomodação em cativeiro destes animais, pois de acordo com Barbosa et al. (2006), esta acomodação pode favorecer a reprodução e prevalência dos ectoparasitos, aumentado assim as chances de infecção, embora, para alguns autores, a transmissão de hemogregarinas por vetores invertebrados em cativeiro seja limitada (Garcia-Navarro \& Pachaly 1994).

Não se podem descartar também outras formas de transmissão, já que a maioria das serpentes infectadas não apresentaram problemas com ectoparasitos, pelo menos no dia do exame clínico e embora não se tenha tido informações sobre o histórico de ectoparasitos das serpentes utilizadas na pesquisa, sabe-se que se tratam de animais vivíparos e que existe a possibilidade de transmissão vertical (Smith 1996), além disso, outros meios de infecção como por mosquitos e pela predação, não podem ser descartadas.

Hawkey \& Denne (1989) descreveram que, na ocorrência de parasitemia há o aumento de linfócitos, basófilos e heterófilos. Garcia-Navarro \& Pachaly (1994) citaram ainda que em infecções por hemogregarinas, pode ocorrer o quadro de anemia hemolítica $(\mathrm{AH})$, fato este não detectado no presente estudo, já que os valores do eritrograma das serpentes positivas encontraram-se dentro da normalidade quando comparados aos valores de referência encontrados por Machado et al. (2006), durante o período de verão em Botucatu/SP. Vale ressaltar que não se encontrou parâmetros de hemograma de serpentes na região Amazônica para tal comparação.

Levando-se em consideração que a contagem das células sanguíneas de répteis varia além de outros fatores, em função da temperatura e como não se encontrou parâmetros de hemograma de serpentes na região Amazônica, os valores de referência utilizados no presente estudo foram os encontrados durante o período de verão por Machado et al. (2006), que estudaram a influência sazonal em valores hematológicos de Boa constrictor amarali mantidas em cativeiro na CEVAP (Centro de Estudos de Venenos de Animais Peçonhentos), Unesp, Campus Botucatu/SP.

Estes resultados são semelhantes àqueles encontrados por Machado et al. (2006), onde de duas serpentes parasitadas por Hepatozoon sp. em seu estudo, nenhuma apresentou alterações hematológicas.

Embora a análise estatística tenha demonstrado não haver relação entre a sanidade das serpentes e a infecção por hemogregarina, sabe-se que em serpentes clinicamente saudáveis o sistema imune age de forma mais precisa, ajudando a inibir infecções, o que explica o fato das duas serpentes que se apresentavam clinicamente doentes somente pela presença de carrapatos, terem manifestados um grau de infecção leve. 
Mesmo que a presença de ectoparasitos tenha se mostrado um fator determinante para a infecção por hemogregarinas, deve-se considerar o fator imunidade atuando no grau da parasitemia destas duas serpentes, já que o mesmo pode ter auxiliado a inibir infecções mais severas.

Vale ressaltar que não se obteve informações a respeito do histórico de ectoparasitos das serpentes do presente estudo, não se sabendo, portanto, se a infecção por hemogregarina se deu pelo contato e desafio anterior a ectoparasitos ou por outras formas de infecção, como a ingestão oral de hospedeiros paratênicos, justificando o fato de algumas das serpentes clinicamente saudáveis encontrarem-se infectadas por hemogregarinas.

De Biase et al. (1989) estudando a presença de hemogregarina em uma serpente Bothrops jararaca mantida em cativeiro no Instituto Butantã, determinaram grau de positividade para Hepatozoon sp. classificado como grave $(+++)$. Após oito anos de cativeiro, este animal foi novamente avaliado e encontrou-se um grau de infecção classificado como leve (+), contando-se raros merozoítos nos esfregaços sanguíneos examinados.

A continuidade da infecção nesta serpente, após este período tão longo, levou os referidos autores a acreditarem na capacidade do hemoparasito em manter-se presente no hospedeiro vertebrado durante longo espaço de tempo, através de várias gerações assexuadas, numa infecção considerada como crônica.

No presente estudo não foi possível realizar a análise da parasitemia nas serpentes em diferentes espaços de tempo, portanto não se pode afirmar se as infecções classificadas como leves podem ser consideradas como infecções crônicas ou não.

\section{CONCLUSÕES}

Foi possível determinar a presença de hemogregarina em Boídeos mantidos em cativeiro no Estado do Pará, tanto em serpentes sadias quanto enfermas.

Não houve correlação entre a presença do hemoparasito, pré-disposição sexual e alterações clínicas e hematológicas importantes nas serpentes hospedeiras, ocorrendo apenas correlação entre a infecção e a presença de ectoparasitos nas mesmas.

Agradecimentos.- Ao Parque Zoobotânico Museu Paraense Emílio Goeldi, em especial ao Dr. Messias Costa.

\section{REFERÊNCIAS}

Barbosa A.R., Silva H., De Albuquerque H.N. \& Ribeiro I.A.M. 2006. Contribuição ao estudo parasitológico de jibóias, Boa constrictor constrictor Linnaeus, 1758, em cativeiro. Revta Biol. Ciênc. Terra 6:1-18.

Campbell T.W. 1996. Clinical pathology, p.248-257. In: Mader D.R. (Ed.), Reptile Medicine and Surgery. W.B. Saunders, Philadelphia.

Cherubini A.L., Barrela T.H. \& Da Silva R.J. 2003. Death of Boa constrictor amarali (Serpentes, Boidae) after ingestion of a tree porcupine (rodentia). J. Venom. Anim. Toxins incl. Trop. Dis. 9:117-124.

Clark K.A., Robinson R.M., Weishuhn L.L., Galvin T.J. \& Horvath K. 1973. Hepatozoon procyonis infections in Texas. J. Wildl. Dis. 9:182-193.

De Biase P., Cardoso Junior R.P. \& Santos S.M. 1989. Presença de Hepatozoon plimeri (Sambon, 1909) - Coccidia, Haemogregarinidae - em exemplar de Bothrops jararaca (Wied, 1824) - serpentes Viperidae, Crotalinae - mantidos em cativeiro. Mem. Inst. Butantan 51:117-120.
Desser S.S., Hong H. \& Martins D.S. 1995. The life history, ultrastructure, and experimental transmission of Hepatozoon catesbianae n. comb., an apicomplexan parasite on the bullfrog, Rana catesbeiana and the mosquito, Culex territans in Algoquin Park, Ontário. J. Parasitol. 81:212-222.

Garcia-Navarro C.E.K. \& Pachaly J.R. 1994. Manual de Hematologia Veterinária. Varela, São Paulo, p.132-133.

Göbel E. \& Krampitz H.E. 1982. Histologische Untersuchungen zur Gamogonie und Sporogonie von Hepatozoon erhardovae in experimentell infizierten Rattenflöhen (Xenopsylla cheopis). Zeitschrift für Parasitenkunde 67:261-271.

Goulart C.E.S. 2004. Hepertologia, Herpetocultura e Medicina de Répteis. L.F. Livros, Rio de Janeiro, p.131-259.

Hawkey C.M. \& Dennet T.B. 1989. A Colour Atlas of Comparative Veterinary Haematology. Wolfe Publishing, London, p.259-275.

Hiinarejos D.P., Hernandez-Divers S.M. \& Hernandez-Divers S.J. 2007. Ofídios, p.126. In: Aguilar R., Hernandez-Divers S.M. \& Hernandez-Divers S.J. (Eds), Atlas de Medicina Terapêutica e Patologia de Animais Exóticos. Interbook, São Paulo.

Hoge A.R. \& Federsoni P.A. 1981. Manutenção de serpentes em cativeiro. Biotério 1:63-73.

Jacobson E. 1986. Parasitic diseases of reptiles, p.162-181. In: Fowler M.E. (Ed.), Zoo and Wild Animal Medicine. W.B. Saunders, Philadelphia.

Kolesnikovas C.K.M., Grego K.F. \& De Albuquerque L.C.R. 2007. Ordem Squamata - Subordem Ophidia (Serpente), p.68-79. In: Cubas Z.S., Silva J.C.R. \& Catão-Dias J.L. (Eds), Tratado de Animais Selvagens: medicina veterinária. Roca, São Paulo.

Lane T.J. \& Mader D.R. 1996. Parasitology, p.185-203. In: Mader D.R. (Ed.), Reptile Medicine and Surgery. W.B. Saunders, Philadelphia.

Leal D.D.M. 2007. Ocorrência de hemoparasitos dos gêneros Trypanosoma (Kinetoplastida: Tripanosomatidae) e hemogregarinas em Anuros dos Estados de São Paulo e Mato Grosso do Sul. Dissertação de Mestrado, Instituto de Biociências, Universidade Estadual Paulista, Botucatu, São Paulo. 47p.

Levine N.D., Corliss J.O., Cox F.E.G., Deroux G., Grain J., Honigberg B.M., Leedale G.F., Loeblich A.R., Lom J., Lynn D., Merinfeld E.G., Page F.C., Poljandky G., Sprague V., Vavra J. \& Wallace F.G. 1980. A newly revised classification of the Protozoa. J. Protozool. 27:37-58.

Machado C.C. 2005. Infecção pelo gênero Hepatozoon em serpentes. Monografia, Universidade Estadual Paulista, Botucatu, SP. 20p.

Machado C.C., Silva L.F.N., Ramos P.R.R. \& Takahira R.K. 2006. Seasonal influence on hematologic values and hemoglobin electrophoresis in Brazilian Boa constrictor amarali. J. Zoo Wildl. Med. 37:487-491.

Moço T.C. 2008. Prevalência de Hepatozoon spp. em serpentes e caracterização morfológica, morfométrica e molecular de Hepatozoon spp. (Apicomplexa, Hepatozoidae) de Crotalus durissus terrificus (Serpentes, Viperidae) naturalmente infectadas. Dissertação de Mestrado, Instituto de Biociências, Universidade Estadual de São Paulo, Botucatu, SP. 67p.

O’Dwyer L.H., Moço T.C., Barrella T.H., Vilela F.C. \& Silva R.J. 2003. Prevalence of Hepatozoon spp. (Apicomplexa, Hepatozoidae) among recently captured Brazilian snakes. Arq. Bras. Med. Vet. Zootec. 55:309-314.

Smith T.G. 1996. The genus Hepatozoon (Apicomplexa: Adeleina). J. Parasitol. 82:565-585.

Telford Jr S.R. 1984. Haemoparasites of reptiles, p.385-517. In: Hoff G.L., Frye F.L. \& Jacobson E.R. (Eds), Diseases of Amphibians and Reptile. Plenum Press, New York.

Telford Jr S.R. 2000. Diagnosis of Reptilian Protozoal Infections, p.243257. In: Fudge A.M. (Ed.) Laboratory Medicine Avian and Exotic Pets. W.B. Saunders, Philadelphia.

Thrall M.A., Baker D.C., Campbell T.W., De Nicola D., Fettman M.J., Lassen E.D., Rebar A. \& Weiser G. 2007. Hematologia e Bioquímica Clínica Veterinária. Roca, São Paulo, p.259-260.

Vanzolini P.E., Costa A.M.M.R. \& Vitt L.J. 1980. Répteis das Caatingas. Academia Brasileira de Ciências, Rio de Janeiro, p.230.

Wosniak E.J., Telford Jr S.R. \& McLaughlin G.L. 1994. Employment of the polymerase chain reaction in the molecular differentiation of reptilian hemogregarines and its application to preventative zoological medicine. J. Zoo. Wildl. Med. 23:538-547. 